\title{
Hole dynamics in a two-dimensional spin-orbit coupled electron system: Theoretical and experimental study of the $\mathrm{Au}(111)$ surface state
}

\author{
I. A. Nechaev, ${ }^{1,2, *}$ M. F. Jensen, ${ }^{3}$ E. D. L. Rienks, ${ }^{3}$ V. M. Silkin, ${ }^{2,4}$ P. M. Echenique, ${ }^{2,4}$ E. V. Chulkov, ${ }^{2,4}$ and Ph. Hofmann ${ }^{3}$ \\ ${ }^{1}$ Department of Theoretical Physics, Kostroma State University, 156961 Kostroma, Russia \\ ${ }^{2}$ Donostia International Physics Center (DIPC), P. de Manuel Lardizabal 4, 20018 San Sebastián, Basque Country, Spain \\ ${ }^{3}$ Institute for Storage Ring Facilities and Interdisciplinary Nanoscience Center, University of Aarhus, 8000 Aarhus C, Denmark \\ ${ }^{4}$ Departamento de Física de Materiales, Facultad de Ciencias Químicas, UPV/EHU and Centro Mixto CSIC-UPV/EHU, Apdo. 1072, \\ 20080 San Sebastián, Basque Country, Spain
}

(Received 29 July 2009; published 16 September 2009)

\begin{abstract}
Using the example of the $\mathrm{Au}(111)$ surface state, the hole inelastic lifetime in a two-dimensional electron system with spin-orbit interaction is investigated within the GW approximation. It is found that taking the spin-orbit interaction into account does not have a significant impact on the surface-state linewidth as a function of energy: for a given binding energy the two spin-orbit-split branches have nearly the same lifetimes. This trend is also observed in angle-resolved photoemission measurements. It is predicted that noticeable differences in the lifetimes of the split branches can be induced by tuning the strength of the splitting and the position of the Fermi level.
\end{abstract}

DOI: 10.1103/PhysRevB.80.113402

The use of the electron's spin instead of its charge for information processing is the basis of spintronics and holds a number of promises, e.g., for fast, low-power devices, and quantum computing. ${ }^{1}$ An important phenomenon in spintronics is the lifting of degeneracies by the spin-orbit interaction (SOI), in particular, for quasi-two-dimensional electron systems which lack inversion symmetry. ${ }^{2-4}$ Recently, this field has witnessed the prediction and discovery of novel effects associated with the SOI, notably the (intrinsic) spin-Hall effect and the quantum spin-Hall effect. ${ }^{5-11}$ An essential ingredient of these effects are carrier transport properties, naturally including inelastic carrier lifetimes. There have been previous speculations ${ }^{12}$ and qualitative observations ${ }^{13}$ that the SOI-induced splitting of bands can severely influence the electron and hole dynamics but quantitative investigations are so far lacking.

In this Brief Report we give a quantitative discussion of the hole lifetimes in a two-dimensional electron system formed by surface-state electrons, where the SOI-induced splitting exists due to the presence of the surface barrier. Using the example of the $\mathrm{Au}(111)$ surface state, we investigate both theoretically (GW calculations) and experimentally [angle-resolved photoemission (ARPES)] how the splitting influences the lifetime of holes in the split bands.

ARPES has revealed a small splitting in the free electronlike surface state of $\mathrm{Au}(111)$, which originates from spinorbit splitting. ${ }^{14-18}$ The electronic structure for this and similar states is sketched in Fig. 1.

The free electronlike parabolic character of the energy dispersion is evident, however the SOI introduces an additional term which is linear in $k$ and results in the splitting into an inner and outer branch [Fig. 1(a)]. The bandwidth of the state can be divided in two regions, I and II. Region I is the energy interval between the bottom of the bands $E_{R}$ and the energy of the band crossing $\epsilon_{0}$. In this region only the outer branch exists, the density of states (DOS) has a singularity at $E_{R}$ [Fig. 1(b)], and a constant energy surface above $E_{R}$ contains two circular features with the spin direction rotating in the same sense [Fig. 1(d)]. Region II covers the
PACS number(s): 71.10.-w, 71.70.Ej, 73.25.+i, 79.60.Bm

binding energy ranging from $\epsilon_{0}$ upward. Here the DOS for the inner and outer branch are different but their sum equals the DOS for the case without SOI. A constant energy surface also shows two circles but with the spin rotating in the opposite directions [Fig. 1(c)].

Alone from these simple DOS considerations, one could expect that the lifetime of a hole at a given binding energy should depend on the branch the hole is in. There is, for example, a larger number states with like spin on the outer branch to fill a hole on the inner branch than vice versa. Within the GW approach, we show, however, that the difference in DOS, along with the factors accounting for the rotation of the spin-quantization axis, ensures equal phase spaces for holes at the same binding energy but in different branches. The remaining, not DOS related, difference between calculated branch lifetimes is small for this particular system, as confirmed by ARPES measurements of the

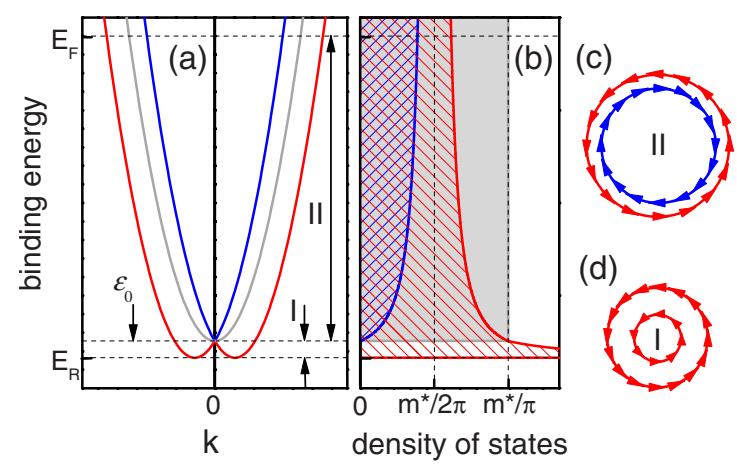

FIG. 1. (Color online) (a) Band dispersion in the quasi-twodimensional system with SOI. The different colors, blue/dark gray and red/gray, denote the inner and outer branches of the spin-orbitsplit dispersion. The light gray curve corresponds to the case without SOI. Regions I and II are explained in text. (b) DOS in the inner (blue/dark gray hatched area) and outer (red/gray hatched area) branch and in the case without the SOI (light gray area). (c) and (d) Constant-energy surfaces with spin directions for regions II and I, respectively. 
$\mathrm{Au}(111)$ surface state linewidth. By tuning the band structure and SOI, however, much stronger differences can be generated.

To make the GW calculations feasible, we use the onedimensional potential model. ${ }^{19}$ We start with the singleparticle states

$$
\psi_{\mathbf{k} n}^{s}\left(z, \mathbf{r}_{\|}\right)=\frac{1}{\sqrt{2}} \varphi_{n}(z) e^{i \mathbf{k} \mathbf{r}_{\|}}\left(\begin{array}{c}
i s e^{-i \phi_{\mathbf{k}}} \\
1
\end{array}\right)
$$

and the corresponding energies

$$
E_{\mathbf{k} n}^{s}=\epsilon_{n}+\frac{\mathbf{k}^{2}}{2 m_{n}^{*}}+s \alpha_{n}|\mathbf{k}| .
$$

Here, $s= \pm$ defines the inner $(+)$ and outer $(-)$ branch for each $n$th band, which is characterized by the effective mass $m_{n}^{*}$, the Rashba parameter $\alpha_{n}$, and the real eigenfunction $\varphi_{n}(z)$ and eigenvalue $\epsilon_{n}$ of a one-dimensional Schrödinger equation. With such a set $\left\{\psi_{\mathbf{k} n}^{s}, E_{\mathbf{k} n}^{s}\right\}$, within the randomphase approximation (RPA), we arrive at the irreducible polarizability

$$
\begin{aligned}
P^{0}\left(z, z^{\prime} ; \mathbf{q}, \omega\right)= & \sum_{n n^{\prime}} \sum_{s s^{\prime}} M_{n n^{\prime}}\left(z, z^{\prime}\right) \int \frac{d \mathbf{k}}{(2 \pi)^{2}} F_{\mathbf{k}, \mathbf{k}+\mathbf{q}}^{s s^{\prime}} \\
& \times \sum_{t= \pm} t \frac{f_{\mathbf{k} n^{\prime}}^{s^{\prime} t} f_{\mathbf{k}+\mathbf{q} n}^{s-t}}{\omega+E_{\mathbf{k} n^{\prime}}^{s^{\prime}}-E_{\mathbf{k}+\mathbf{q} n}^{s}+i t \eta}
\end{aligned}
$$

where $f_{\mathbf{k} n}^{s+}=f_{\mathbf{k} n}^{s}$ and $f_{\mathbf{k} n}^{s-}=1-f_{\mathbf{k} n}^{s}$ with $f_{\mathbf{k} n}^{s}$ being Fermi factors, $M_{n n^{\prime}}\left(z, z^{\prime}\right)=\varphi_{n}(z) \varphi_{n}\left(z^{\prime}\right) \varphi_{n^{\prime}}(z) \varphi_{n^{\prime}}\left(z^{\prime}\right), \quad$ and $\quad F_{\mathbf{k}, \mathbf{k}+\mathbf{q}}^{s s^{\prime}}=\frac{1}{2}[1$ $\left.+s s^{\prime} \cos \left(\phi_{\mathbf{k}}-\phi_{\mathbf{k}+\mathbf{q}}\right)\right]$ the mentioned factors reflecting the rotations in spin space. The obtained $P^{0}$ determines the RPAscreened interaction $W$ through the standard relation (see, e.g., Ref. 20). This screened interaction, in turn, allows us to calculate the GW self-energy matrix elements $\left\langle\psi_{\mathbf{k} n}^{s}|\Sigma(\omega)| \psi_{\mathbf{k} n}^{s}\right\rangle$. For holes $\left(\omega<E_{F}\right)$, the imaginary part of these elements reads as

$$
\begin{aligned}
\operatorname{Im}\left\langle\psi_{\mathbf{k} n}^{s}|\Sigma(\omega)| \psi_{\mathbf{k} n}^{s}\right\rangle= & -\sum_{n^{\prime} s^{\prime}} \int d z d z^{\prime} M_{n n^{\prime}}\left(z, z^{\prime}\right) \\
& \times \int \frac{d \mathbf{q}}{(2 \pi)^{2}} F_{\mathbf{k}, \mathbf{q}}^{s s^{\prime}} \mathbf{q}_{\mathbf{q} n^{\prime}}^{s^{\prime}} \theta\left(E_{\mathbf{q} n^{\prime}}^{s^{\prime}}-\omega\right) \\
& \times \operatorname{Im} W\left(z, z^{\prime} ; \mathbf{k}-\mathbf{q}, \omega-E_{\mathbf{q} n^{n^{\prime}}}^{s^{\prime}}\right),
\end{aligned}
$$

where $\theta(x)$ is the step function. Finally, the inverse lifetime (the linewidth) is defined as $\Gamma_{\mathbf{k} n}^{s}=2\left|\operatorname{Im}\left\langle\psi_{\mathbf{k} n}^{s}\left|\Sigma\left(E_{\mathbf{k} n}^{s}\right)\right| \psi_{\mathbf{k} n}^{s}\right\rangle\right|$. Since we are interested in the surface-state linewidth, we examine $\Gamma_{\mathbf{k} n}^{s}$ for $n=0$ that in our calculations corresponds to the surface state. To reproduce the $\mathrm{Au}(111)$ surface band structure, we take $\alpha_{0}=3.5118 \times 10^{-11} \mathrm{eV} \mathrm{m},{ }^{15,16} m_{0}^{*}=0.28$, and $\epsilon_{0}=-474.5 \mathrm{meV} .{ }^{20} \mathrm{~A}$ variation in these parameters within the spread that exists in the literature has no visible effect on final results. For $n$ corresponding to the bulk occupied states, $\alpha_{n}=0$, and the effective mass $m_{n}^{*}$ is band dependent, changing gradually from 0.21 at the gap edge to unity for deeper bulk states. ${ }^{20}$

Figure 2(a) shows the resulting $\Gamma_{\mathbf{k} 0}^{s}$ as a function of mo-

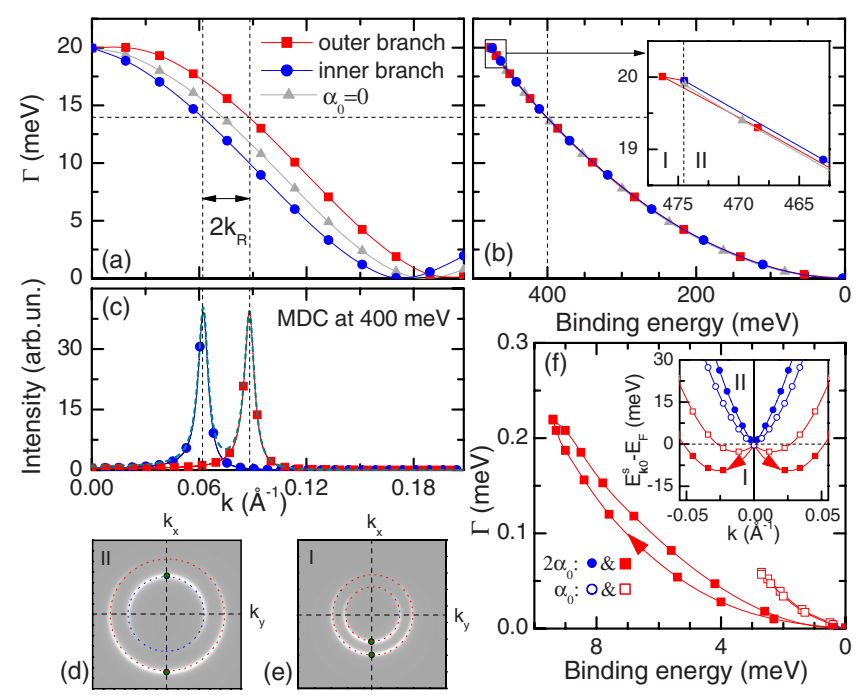

FIG. 2. (Color online) (a) Calculated $|\mathbf{k}|$ dependence of the $\mathrm{Au}(111)$ surface-state linewidth $\Gamma_{\mathbf{k} 0}^{s}\left(\alpha_{0}=0\right.$ corresponds to the case without the SOI). (b) The same linewidth as a function of binding energy. Inset: magnification of the linewidth data in the vicinity of the border between region I and II. (c) Modeled MDC (separately for each branch and in sum) at a binding energy of $400 \mathrm{meV}$. (d) and (e) Sketch of $F_{\mathbf{k}, \mathbf{q}}^{s s^{\prime}}$ entering Eq. (4) on a constant-energy surface for regions II and I, respectively. (f) Calculated energy dependence of $\Gamma_{\mathbf{k} 0}^{s}$ for a hypothetical surface state with $E_{F} \approx \epsilon_{0}$ and the $\mathrm{Au}(111)$ Rashba parameter $\alpha_{0}$ (open circles and squares) as well as $2 \alpha_{0}$ (filled circles and squares). Inset: band dispersion of the surface state for the two cases.

mentum for both branches of the spin-orbit-split surface state. Originating from the same point at $k=0$, the curves $\Gamma_{\mathbf{k} 0}^{+}$ and $\Gamma_{\mathbf{k} 0}^{-}$show practically the same momentum dependence with a shift of $2 k_{R}=2 m_{0}^{*} \alpha_{0}$, as expected from the relationship $E_{\left(1+2 k_{R} / k\right) \mathbf{k} 0}^{-}=E_{\mathbf{k} 0}^{+}$. Using Eq. (2) the $k$-dependent $\Gamma_{\mathbf{k} 0}^{s}$ can be converted into a energy-dependent one which is shown in Fig. 2(b). In this way it can be seen that the linewidth shows a negligible branch dependence. We have found the deviations to be within $\sim 1 \%$, the largest of which occur in the energy range shown in the inset of Fig. 2(b).

The behavior of $\Gamma_{\mathbf{k} 0}^{s}$ should be observable in the linewidth of momentum distribution curves (MDC) measured by ARPES. ${ }^{21}$ An MDC is a $\mathbf{k}$ cut through the spectral function

$$
A_{0}(\mathbf{k}, \omega)=\frac{1}{\pi} \sum_{s} \frac{\Gamma_{\mathbf{k} 0}^{s} / 2}{\left[\omega-E_{\mathbf{k} 0}^{s}\right]^{2}+\left[\Gamma_{\mathbf{k} 0}^{s} / 2\right]^{2}} .
$$

at a fixed energy $\omega$. We expect peaks at the $\mathbf{k}$ values corresponding to the dispersion of the inner and outer branches. The linewidth $\Gamma_{\mathbf{k} 0}^{s}$ in the vicinity of these $\mathbf{k}$ values gives the $k$ widths of the peaks. The dashed lines crossing Figs. 2(a)-2(c) indicate that the $k$ widths of the peaks of both contributions should be practically the same. In the total MDC the inner peak can appear to be higher and wider. This is also observed in the experiment and the reason is discussed below. As to region I, the splitting for $\mathrm{Au}(111)$ is so small that the peaks cannot be resolved. 

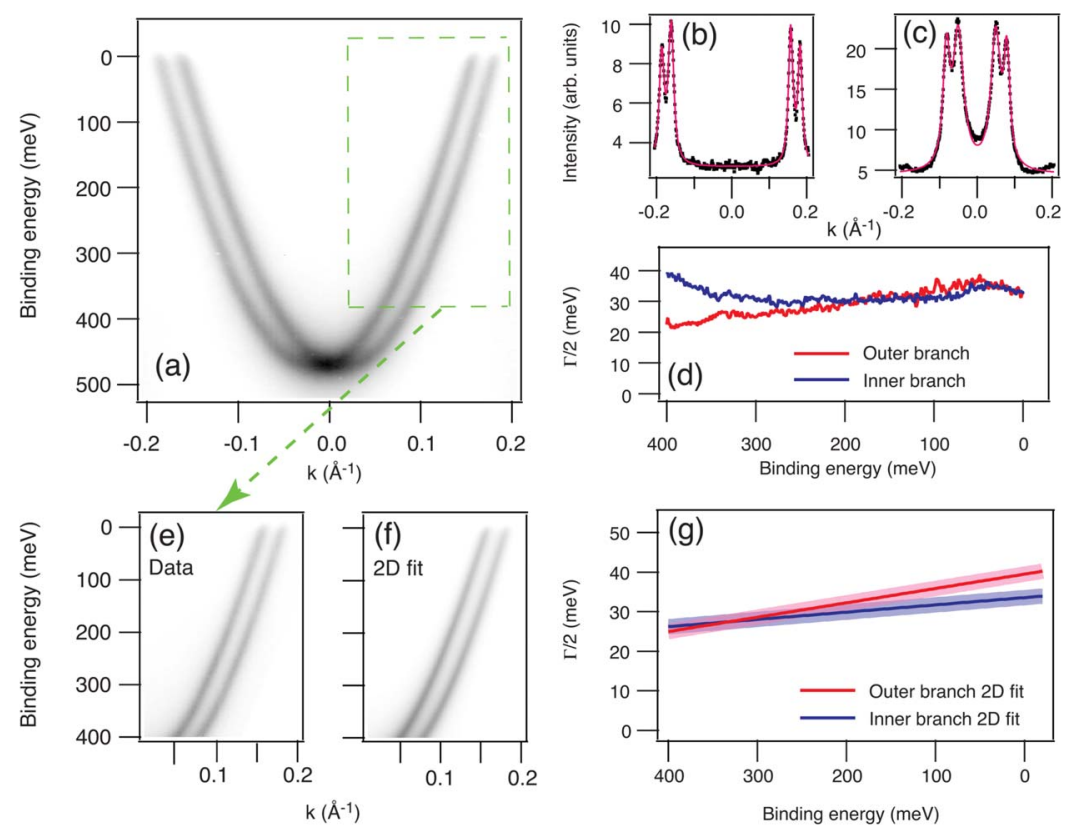

FIG. 3. (Color online) (a) Photoemission intensity from the $\mathrm{Au}(111)$ surface state $(h \nu$ $=16 \mathrm{eV}$ ). (b) and (c) MDC cuts through the data in (a) at binding energies of 0 and $400 \mathrm{meV}$, respectively, together with a fit to the data. (d) Linewidth from fits as in (b) and (c). (e) Magnification of the data in (a). (f) Result from a fit of a two-dimensional calculated spectral function to the data shown in (e). (g) Linewidth from the two-dimensional fit shown in (f). Light-colored areas represent the uncertainties.
The obtained results can be understood if one notes that Eq. (4) differs from the spin-degenerate case by the factors $F_{\mathbf{k}, \mathbf{q}}^{s s^{\prime}}$ and the split energy dispersion. The factors govern possible scattering processes while the splitting causes different transition momenta to be involved in intrabranch and interbranch scattering. Figure 2(d) shows that in region II a hole on the top of the inner circle or a hole on the bottom of the outer circle can be filled by the electrons from states of the bright areas at the energy in question and from the similar areas at higher energies up to $E_{F}$. Taking into account the densities of such states [see Fig. 1(b)], we can infer that the resulting phase space for both branches is the same and equal to that in the nonsplit case. Remaining differences in linewidths can solely arise from the splitting through the $\mathbf{k}$ dependence of $\operatorname{Im} W(\mathbf{k}, \omega)$. In the SOI-induced energy region I, which contains the outer branch only, the difference between lifetimes of holes in the inner and outer circle [Fig. 2(e)] is also caused by the mentioned $\mathbf{k}$ dependence. An analysis has shown that for both regions the difference grows with increasing $\alpha_{0}$. Moreover, at a fixed Rashba parameter the difference caused by the splitting becomes significant when the region I is approaching the Fermi level. Figure 2(f) represents our results for an extreme situation of the hypothetical surface state with $E_{F} \approx \epsilon_{0}$. Note that for such a system one can effectively tune the lifetime through the Rashba parameter by changing an applied electric field.

The theoretical predictions for region II can be verified by ARPES. In particular, it should be possible to check if the two branches of the dispersion have practically the same width at a given energy for the Au(111) surface. ARPES data were taken at the SGM-3 beamline of the synchrotron radiation facility ASTRID in Aarhus. ${ }^{22}$ The combined energy resolution of beamline and analyzer was better than $10 \mathrm{meV}$, the experimentally determined $k$ resolution was $0.004 \AA^{-1}$. The sample temperature was $\sim 60 \mathrm{~K}$. The $\mathrm{Au}(111)$ surface was prepared by standard methods. ${ }^{14,15,23}$

Figure 3(a) shows the photoemission intensity from the $\mathrm{Au}(111)$ surface state. The data reported here compare well to earlier findings. ${ }^{15,23}$ The linewidth of the surface state in an energy distribution curve through $\bar{\Gamma}$ is $48 \mathrm{meV}$ and thereby somewhat larger than the lowest reported value (at $T=30 \mathrm{~K}$ ) of $27 \mathrm{meV}{ }^{15}$ The difference is attributed to the higher temperature and defect scattering due to surface imperfections. The two branches of the dispersion, on the other hand, are better resolved than in Ref. 15, due to the higher $k$ resolution here.

Figures 3(b) and 3(c) show MDC fits at two different binding energies: 0 and $400 \mathrm{meV}$. For the fit a Voigt function was employed to account for the finite $k$ resolution. Figure 3(d) shows the resulting energy-dependent linewidth for the two branches obtained from the MDC fits and the dispersion. At low binding energies $(<200 \mathrm{meV})$ both branches have approximately the same linewidth but their absolute value is higher than the theoretical value. This can be explained by phonon and defect-mediated decay, neither of which is included in the calculation. In the present case both are expected to be approximately independent of energy. Both branches are also found to be slightly narrower at higher binding energies, a well-known effect in ARPES in the presence of defect scattering. ${ }^{24,25}$ At binding energies $>200 \mathrm{meV}$, pronounced differences between the branches are observed. The outer branch follows the expected trend but the inner branch broadens significantly. This behavior disagrees qualitatively with the theoretical prediction.

The cause for the disagreement is that the usual approach to ARPES data analysis is not well suited here. So far, we have tacitly assumed that an MDC cut through the spectral function can be treated as a sum of Lorentzian lines, Eq. (5), permitting the extraction of the linewidth. Strictly spoken this is only correct for a linear dispersion of the state and invalid near the bottom of the band. This can already be seen in the fits of Figs. 3(b) and 3(c). Near the Fermi energy the data can be fitted well but at $400 \mathrm{meV}$ the fit is visibly poorer, especially for the inner two peaks and the background between them. The reason is the finite energetic width of the states near the bottom of the band which in- 
creases the background in the center of an MDC taken at higher binding energies.

This qualitatively explains the poor fit at higher binding energies but it does not allow us to decide if the different linewidths are solely caused by this effect. To this end, we devise an alternative way of analyzing ARPES data which goes beyond the well-established approach of fitting onedimensional MDCs. Rather, we attempt a two-dimensional fit to a measured image with a resolution-broadened spectral function, constructed of two branches with the form (5). This spectral function is entirely described by the state's dispersion, intensity, and self-energy. The energy-dependent intensity and the dispersion are higher-order polynomials with parameters left free in the fit, in order to account for nonparabolicity, image distortions, and an energy dependence of the photoemission matrix element and analyzer transmission function. For simplicity, we assume a linear energy dependence for the self-energy, i.e., the linewidth. This twodimensional fitting scheme turns out to be very stable because of the large number of data points and the relatively small number of parameters.

Figures 3(e) and 3(f) show the subset of the data used for the fit and the result of the fit, respectively. Apart from the noise in the data, they are virtually indistinguishable. The most important result is that the line half widths in Fig. 3(g) are very similar for both branches. This result confirms that the difference seen in the simple analysis using MDCs, Fig. 3(d), is an artifact. We can therefore conclude that there is no experimental indication for a difference in lifetime between the two branches. Moreover, this result indicates that the theoretical prediction of negligible difference between branches of $\mathrm{Au}(111)$ also holds in the presence of electronphonon and electron-defect scattering.

In conclusion, we have theoretically discussed the effect of spin-orbit interaction on the lifetimes in a twodimensional electron system and have illustrated this by the case of the $\mathrm{Au}(111)$ surface, that is a system dominated by the region II in the dispersion. We have demonstrated that at a given binding energy the hole lifetime is practically independent on the branch of the spin-orbit-split surface state. This is confirmed by ARPES data from $\mathrm{Au}(111)$ but only if a fitting procedure is employed which directly analyzes the two-dimensional spectral function rather than cuts through it. We have also shown that the difference between the linewidths of the two branches can arise solely from the $\mathbf{k}$ dependence of $\operatorname{Im} W(\mathbf{k}, \omega)$. For a region I dominated system, noticeable deviations in the lifetime are predicted and it can be expected that applied electric fields would not only influence the size of the splitting but also the lifetime of the states in different branches. The effects predicted here should be observable in systems with much stronger splitting which pushes states toward the Brillouin-zone boundary ${ }^{12}$ or in cases where the SOI and occupation can be designed at will. $^{26}$

We acknowledge support from the Lundbeck Foundation, the Danish National Research Council, the University of the Basque Country (Grant No. GIC07IT36607), the Departamento de Educación del Gobierno Vasco, and the Spanish Ministerio de Ciencia y Tecnología (MCyT) (Grant No. FIS200766711C0101). The work of V.M.S. is sponsored by the Ikerbasque foundation.
*Also at REC "Physics and Chemistry of High-Energy Systems", Tomsk State University, 634050, Tomsk, Russia.

${ }^{1}$ I. Zutic, J. Fabian, and S. Das Sarma, Rev. Mod. Phys. 76, 323 (2004).

${ }^{2}$ E. I. Rashba, Sov. Phys. Solid State 2, 1109 (1960).

${ }^{3}$ Y. A. Bychkov and E. I. Rashba, J. Phys. C 17, 6039 (1984).

${ }^{4}$ Y. A. Bychkov and E. I. Rashba, JETP Lett. 39, 78 (1984).

${ }^{5}$ S. Murakami, N. Nagaosa, and S.-C. Zhang, Science 301, 1348 (2003).

${ }^{6}$ J. Sinova, D. Culcer, Q. Niu, N. Sinitsyn, T. Jungwirth, and A. MacDonald, Phys. Rev. Lett. 92, 126603 (2004).

${ }^{7}$ Y. Kato, R. Myers, A. Gossard, and D. Awschalom, Science 306, 1910 (2004).

${ }^{8}$ C. L. Kane and E. J. Mele, Phys. Rev. Lett. 95, 146802 (2005).

${ }^{9}$ B. A. Bernevig, T. L. Hughes, and S.-C. Zhang, Science 314, 1757 (2006).

${ }^{10}$ B. A. Bernevig and S.-C. Zhang, Phys. Rev. Lett. 96, 106802 (2006).

${ }^{11}$ M. Konig, S. Wiedmann, C. Brune, A. Roth, H. Buhmann, L. W. Molenkamp, X.-L. Qi, and S.-C. Zhang, Science 318, 766 (2007).

${ }^{12}$ Y. M. Koroteev, G. Bihlmayer, J. E. Gayone, E. V. Chulkov, S. Blügel, P. M. Echenique, and P. Hofmann, Phys. Rev. Lett. 93, 046403 (2004).

${ }^{13}$ J. I. Pascual et al., Phys. Rev. Lett. 93, 196802 (2004).

${ }^{14}$ S. LaShell, B. A. McDougall, and E. Jensen, Phys. Rev. Lett. 77,
3419 (1996).

${ }^{15}$ F. Reinert, G. Nicolay, S. Schmidt, D. Ehm, and S. Hüfner, Phys. Rev. B 63, 115415 (2001).

${ }^{16}$ F. Reinert, J. Phys.: Condens. Matter 15, S693 (2003).

${ }^{17}$ J. Henk, A. Ernst, and P. Bruno, Phys. Rev. B 68, 165416 (2003).

${ }^{18}$ M. Hoesch, M. Muntwiler, V. N. Petrov, M. Hengsberger, L. Patthey, M. Shi, M. Falub, T. Greber, and J. Osterwalder, Phys. Rev. B 69, 241401(R) (2004).

${ }^{19}$ E. V. Chulkov, V. M. Silkin, and P. M. Echenique, Surf. Sci. 437, 330 (1999).

${ }^{20}$ V. M. Silkin, J. M. Pitarke, E. V. Chulkov, and P. M. Echenique, Phys. Rev. B 72, 115435 (2005).

${ }^{21}$ C. Kirkegaard, T. K. Kim, and P. Hofmann, New J. Phys. 7, 99 (2005).

${ }^{22}$ S. V. Hoffmann, C. Søndergaard, C. Schultz, Z. Li, and P. Hofmann, Nucl. Instrum. Methods Phys. Res. A 523, 441 (2004).

${ }^{23}$ S. LaShell, B. A. McDougall, and E. Jensen, Phys. Rev. B 74, 033410 (2006).

${ }^{24}$ S. D. Kevan, Phys. Rev. B 33, 4364 (1986).

${ }^{25}$ A. Beckmann, K. Meinel, M. Heiler, C. Ammer, and H. Neddermeyer, Phys. Status Solidi B 198, 665 (1996).

${ }^{26}$ C. R. Ast, D. Pacile, L. Moreschini, M. C. Falub, M. Papagno, K. Kern, M. Grioni, J. Henk, A. Ernst, S. Ostanin, and P. Bruno, Phys. Rev. B 77, 081407(R) (2008). 